\title{
X-rays from Star-Forming Regions in the VLT Era
}

\author{
Thierry Montmerle ${ }^{1}$ and Nicolas Grosso ${ }^{2}$ \\ 1 Service d'Astrophysique, CEA Saclay, 91191 Gif-sur-Yvette, France \\ 2 Max-Planck Institut für Extraterrestrische Physik, D-85741 Garching, Germany
}

\begin{abstract}
The association between star-forming regions and X-ray emission was discovered over 30 years ago. We now know that essentially all young stellar objects, $\mathrm{T}$ Tauri stars and protostars, are X-ray emitters, although the case of the youngest, Class 0 protostars, is less clear. The paper highlights X-ray emission and absorption mechanisms, and summarizes X-ray observations of young stellar objects. The impact of the VLT on the characterization of the new X-ray sources is also briefly discussed.
\end{abstract}

\section{From past to present}

The first evidence that X-rays could reach the Earth from outer space, and more specifically from the Sun, was obtained in 1949 by means of a V2 rocket launched from White Sands, New Mexico, by a team of physicists from the US Naval Research Laboratory; the flight lasted a mere 336 s (Friedman, Lichtman, \& Byram 1951). The real astronomical start took place with the launch of the Uhuru satellite, in 1970. This satellite, operating for three years in scanning mode with collimators, obtained the first X-ray all-sky survey, with the discovery of 339 sources, with error boxes up to several $10^{\prime}$ on a side. One of them included M42, the Orion nebula (Giacconi et al. 1972): the knowledge that star-forming regions are emitting $X$-rays is nearly 30 years old! The discovery of the X-ray emission from M42 was confirmed by the ANS satellite (den Boggende 1978). The second major leap was the launch of the second "High-Energy Astronomical Observatory", Einstein, in 1978, which carried X-ray focussing mirrors able to obtain the first pointed images of astronomical X-ray sources, with a field-of-view of $\sim 1^{\circ} \times 1^{\circ}$. Orion and other star-forming regions were successfully detected, with a combination of point sources identified with $\mathrm{OB}$ stars and/or T Tauri stars (Ku \& Chanan 1979). Launched in 1990, ROSAT undertook an all-sky survey, which resulted in a catalog of several $10^{5}$ sources, including $\sim 50,000$ stars in all evolutionary stages, from PMS to evolved. Launched in 1993, the Japanese satellite ASCA was the first imaging satellite using CCD detectors and reaching the hard X-ray range. To this date, four X-ray satellites are in orbit: BeppoSAX, RossiXTE, and the two "workhorses" of the present decade, the US Chandra and the European XMM-Newton, both launched in 1999. These two major observatories currently have significant programs dedicated to the study of young clusters and star-forming regions. Tables 1 and 2 give the current list of accepted targets, updated from Sciortino (2001). 
Table 1. SFRs and young stellar clusters in the Chandra AO-1 Garanteed Time Observation (GTO) and Guest Observation (GO) AO-1, AO-2 [left] and AO-3 [right] programs (June 2001)

\begin{tabular}{ll|ll}
\hline Target Name & Exposure Time & Target Name & Exposure Time \\
\hline NGC 1333 & $50 \mathrm{ks}$ & IRAM04191 & $20 \mathrm{ks}$ \\
IC 348 & $50 \mathrm{ks}$ & L1527 & $20 \mathrm{ks}$ \\
Orion Trapezium & $50 \mathrm{ks}+69 \mathrm{ks}$ & $\alpha$ Tau & $20 \mathrm{ks}$ \\
Orion region & several obs & ONC Flanking Field S $50 \mathrm{ks}$ \\
R CrA Cloud Core & $20 \mathrm{ks}$ & ONC Flanking Field N $50 \mathrm{ks}$ \\
$\rho$ Oph & mosaic of $6 \times 5 \mathrm{ks}$ & $\sigma$ Ori & $100 \mathrm{ks}$ \\
$\rho$ Oph & $2 \times 100 \mathrm{ks}$ & HH 24-26 & $70 \mathrm{ks}$ \\
NGC2516 & $20 \mathrm{ks}+20 \mathrm{ks}$ & Maddalena's Cloud & $30 \mathrm{ks}$ \\
NGC2516 & $20 \mathrm{ks}+50 \mathrm{ks}$ & NGC2264 & $50 \mathrm{ks}+100 \mathrm{ks}$ \\
M16 & $80 \mathrm{ks}$ & NGC2362 & $47 \mathrm{ks}+48 \mathrm{ks}$ \\
NGC 6530 & $61 \mathrm{ks}$ & IRAS16293 & $30 \mathrm{ks}$ \\
W3B & $40 \mathrm{ks}$ & Trifid Nebula & $60 \mathrm{ks}$ \\
HH 1 & $20 \mathrm{ks}$ & HH 80/81 & $40 \mathrm{ks}$ \\
\hline
\end{tabular}

Table 2. SFRs and young stellar clusters in the XMM-Newton/EPIC GTO and AO-1 GO program (June 2000).

\begin{tabular}{ll|ll}
\hline Target Name & Exposure Time & Target Name Exposure Time \\
\hline NGC 2024 & $45 \mathrm{ks}+25 \mathrm{ks}$ & L1448-C & $30 \mathrm{ks}$ \\
NGC 2023 & $25 \mathrm{ks}$ & NGC 1333 & $50 \mathrm{ks}$ \\
IC 2602 & $45 \mathrm{ks}$ & Orion P1795 & $20 \mathrm{ks}$ \\
IC 2391 & $45 \mathrm{ks}$ & OMC2 $/ 3$ & $100 \mathrm{ks}$ \\
alpha Per & $50 \mathrm{ks}$ & L1641-N & $50 \mathrm{ks}$ \\
Upper Sco-Cen $2 \times 50 \mathrm{ks}$ & NGC 2264 & $2 \times 35 \mathrm{ks}$ \\
R CrA & $20 \mathrm{ks}$ & Chamaeleon & $28 \mathrm{ks}$ \\
$\rho$ Oph & $25 \mathrm{ks}+50 \mathrm{ks}$ & Serpens & $50 \mathrm{ks}$ \\
IC 348 & $40 \mathrm{ks}$ & NGC 2362 & $50 \mathrm{ks}$ \\
L1551 & $50 \mathrm{ks}$ & NGC 2547 & $50 \mathrm{ks}$ \\
Blanco 1 & $50 \mathrm{ks}$ & Pleiades & $2 \times 50 \mathrm{ks}$ \\
\hline
\end{tabular}

The latest review of pre-Chandra/XMM-Newton results on Young Stellar Objects (YSOs) is by Feigelson \& Montmerle 1999 (FM); the review by Glassgold, Feigelson \& Montmerle (2001; hereafter GFM) puts more emphasis on the various physical processes at work in YSOs and in their vicinity. The reader is referred to these reviews for details. In the present short contribution, we only give some key background information, summarize and update the main X-ray results, and emphasize the role of the VLT in the problem of the characterization of the new X-ray sources. 


\section{X-ray emission and absorption mechanisms}

- YSO X-ray emission comes from the thermal bremsstrahlung emission of an optically thin, "coronal" plasma at temperatures $T_{\mathrm{X}} \sim 10^{6}-10^{8} \mathrm{~K}$ and densities $n_{\mathrm{e}} \sim 10^{10}-10^{12} \mathrm{~cm}^{-3}(\mathrm{FM})$. It can be shown that the emitting plasma must be confined in magnetic loops, like on the Sun, but much larger. Stellar X-ray spectra also indicate the presence of lines of heavy elements (see examples in, e.g., Audard et al. 2001), so that abundances and metallicities can be determined from $\chi^{2}$-type fits. This is also the case for the X-ray brightest young stars.

- $X$-ray absorption is an important parameter in all studies of young stars (GFM). It is due to the photoelectric effect, in which inner-shell (mostly K and L) electrons of an atom are ejected by the incoming photon. However, these photoelectrons basically carry away the energy of the original X-rays (in the keV range), minus the shell energy, so are themselves initially highly energetic compared to the energy of the outer shells of the ambient atoms. A "shower" results, in which many secondary electrons are ejected from ambient atoms as the primary electrons collide with them and loose their initial energy. Other secondary electrons may be produced as "Auger electrons", i.e., from internal rearrangement of the energy levels of the original atoms hit by the X-ray photons.

Fig. 1, adapted from Ryter (1996), gives in graphic form the total extinction cross-section all the way from the far-IR to the hard X-ray range, assuming a normal gas-to-dust ratio and cosmic abundances. First, it can be noted that the cross-section has comparable values in the IR range (where it is dominated by the dust) and in the $X$-ray range (where it is dominated by the gas): in other words, the "penetrating power" of X-rays is the same as in the IR, a key point allowing detailed studies of embedded sources such as young T Tauri stars (hereafter TTS) and protostars (see the detailed discussion by Casanova et al. 1995). Second, the total photoelectric cross-section, which for each element has the form $\sigma \propto E_{\mathrm{X}}^{-2.5}$ beyond the Lyman limit $(\mathrm{H})$, displays discontinuities. With increasing X-ray energy, each "jump" corresponds to the inner-shell ionization of a new atomic species present in gaseous form in the ISM: He, C, N, O below $1 \mathrm{keV}$, up to Fe and Ni above $\sim 7 \mathrm{keV}$. Although these cross-section jumps generally are small, they accumulate to the point that above $0.5 \mathrm{keV}$ the absorption comes predominantly from heavy atoms, and is $>10$ times larger than from $\mathrm{H}+\mathrm{He}$ beyond $\sim 7 \mathrm{keV}$ (for details, see Wilms, Allen, and McCray 2000).

For $E_{X}=1 \mathrm{keV}$ and solar abundances, one finds $\tau(1 \mathrm{keV})=N_{\mathrm{H}} / 4.41 \times$ $10^{21} \mathrm{~cm}^{-2}$. If the usual conversion between $N_{\mathrm{H}}$ and $A_{\mathrm{V}}$ for diffuse interstellar clouds is used (e.g., Ryter 1996), then $\tau(1 \mathrm{keV})=1$ occurs for $A_{\mathrm{V}} \simeq 2$. It is

also a useful rule-of-thumb that the penetrability of X-rays varies like $\sim E_{\mathrm{X}}^{-2.5}$ : the absorption decreases by a factor $\sim 3000$ between $0.4 \mathrm{keV}$ and $10 \mathrm{keV}$. Note that the contribution of dust grains (into which certain heavy atomic species are condensed) to the X-ray opacity is small, and depends on grain models only at the lowest energies $\left(E_{\mathrm{X}} \lesssim 0.3 \mathrm{keV}\right.$ ) (see discussion in Wilms, Allen, and McCray 2000). 


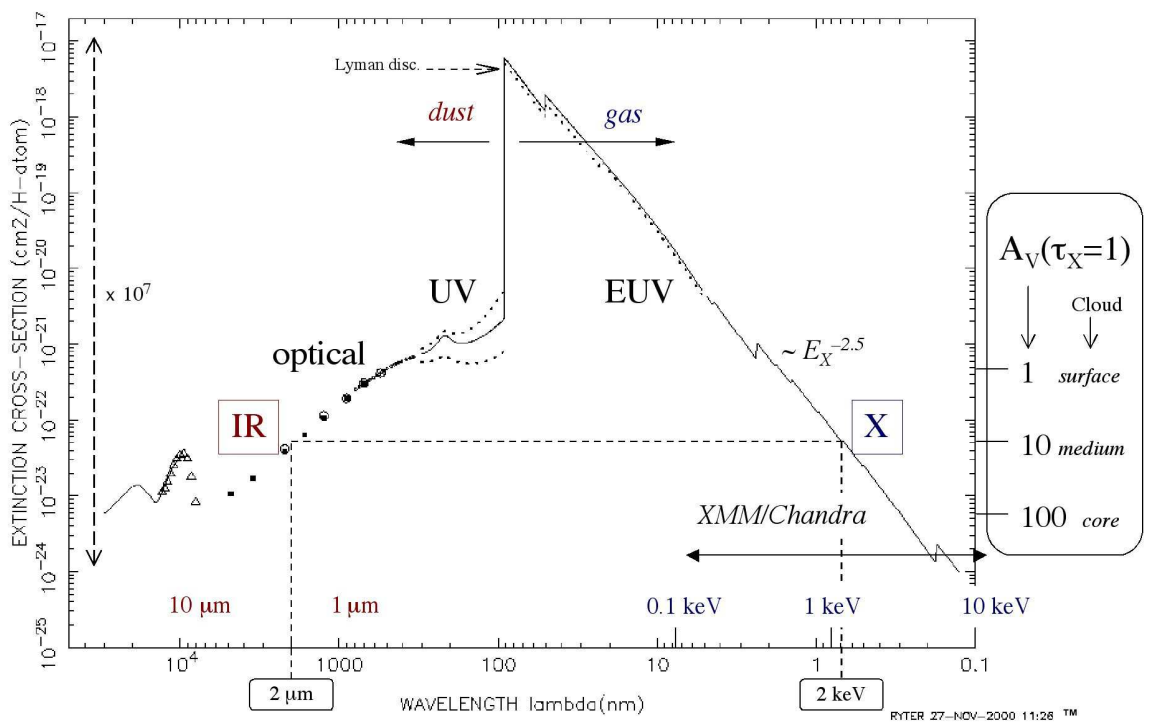

Fig. 1. Total extinction cross section, from the IR range $(1 \mu \mathrm{m} \equiv 1000 \mathrm{~nm})$, to the $\mathrm{X}$-ray range $\left(1 \mathrm{keV} \equiv 0.81 \mathrm{~nm}\right.$, using $E=h \times \lambda^{-1}$, with $h$ the Planck's constant) (see Ryter 1996). The sharp peak in the middle is the Lyman discontinuity (almost four orders of magnitude!).

\section{X-ray observations of low-mass star-forming regions}

To date, all major star-forming regions within $\sim 500 \mathrm{pc}$ of the Sun have been observed in X-rays, plus a couple of more distant ones $(d \geq 1 \mathrm{kpc})$ (see FM). Perhaps the most spectacular observation to date is that of the Orion Trapezium region and its surrounding M42 nebula (Garmire et al. 2000), in which a single $17^{\prime} \times 17^{\prime}$ Chandra field has revealed nearly 1,000 sources. As shown on Fig. 2 , almost every X-ray source has a stellar counterpart (position accuracy $\sim 0.1^{\prime \prime}$ ), all the way from the central massive stars down to lower-mass stars. A full analysis is in progress (Feigelson et al., in preparation).

Here we restrict our discussion to the numerous X-ray detected low-mass, solar-like stars. The Taurus-Auriga, R Coronae Australis, Chamaeleon, and $\rho$ Ophiuchi regions have been particularly well studied for many years. For example, ROSAT observations of the central region of the $\rho$ Ophiuchi dark cloud have revealed several dozen X-ray sources associated with young stellar objects (Grosso et al. 2000). This region has now been also observed in X-rays by Chandra (Imanishi et al. 2001), and by XMM-Newton (Grosso et al., in preparation: see Fig. 3). ROSAT being sensitive in the soft X-ray band only $(<2.4 \mathrm{keV})$, the sources are mainly identified with objects having low to intermediate extinction (see Fig. 1), i.e., TTS. Nevertheless thanks to X-ray flares, which combine an 


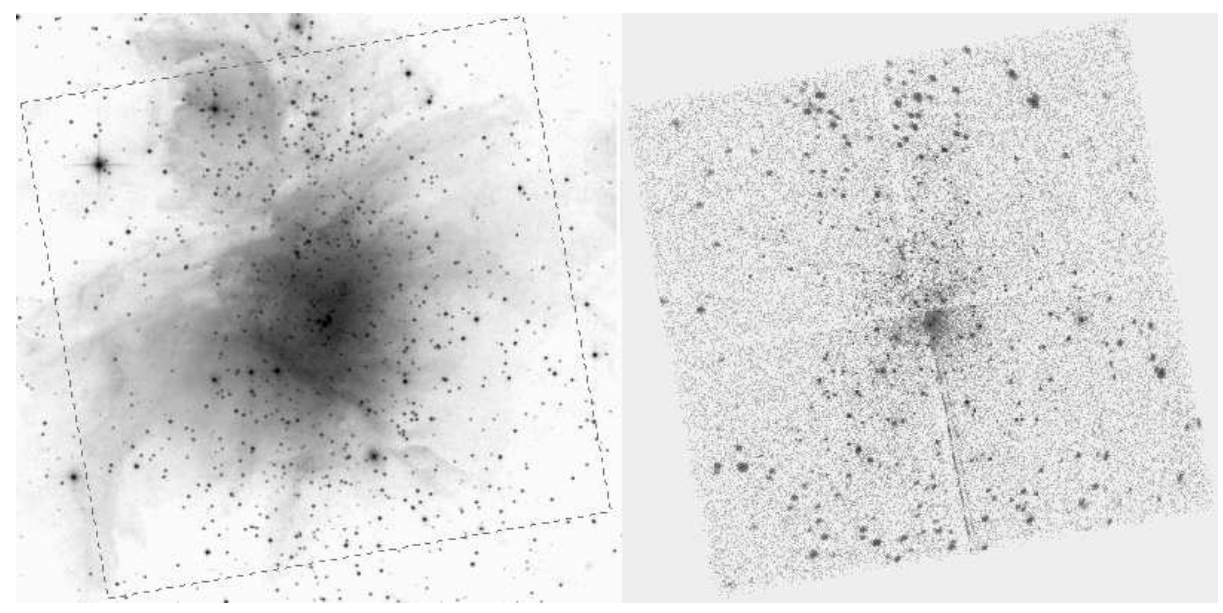

Fig. 2. Left: The Orion Nebula Cluster seen at $2 \mu \mathrm{m}$, from the $2 \mathrm{MASS}$ survey. The Trapezium O stars exciting the M42 nebula are located at the center of the image. Right: The same region seen by Chandra/ACIS. Almost $1000 \mathrm{X}$-ray sources are detected (Garmire et al. 2000); there is nearly a one-to-one subarcsecond correspondence between the near-IR and X-ray sources in both images. Field of view of Chandra/ACIS: $17^{\prime} \times 17^{\prime}$.

increase of X-ray luminosity with an increase of plasma temperature hence a better visibility, ROSAT caught X-rays from two protostars (see Grosso et al. 1997 and Grosso 2001). In contrast ASCA was sensitive up to $10 \mathrm{keV}$, hence was able to penetrate deep into dense material, revealing more easily X-rays from protostars. The past generation of X-ray satellites detected protostars only during their high activity level. Today, the new generation of X-ray satellites, Chandra and XMM-Newton, with an increased sensitivity up to $10 \mathrm{keV}$, gives access to the quiet coronae of protostars.

Let us now summarize and update briefly some key X-ray properties of TTS and protostars.

- There is a strong correlation between the X-ray and bolometric luminosities of TTS, which differs somewhat from region to region: $L_{\mathrm{X}} / L_{\mathrm{bol}} \sim 10^{-4}$, as compared with $\sim 10^{-6}$ for the active Sun. Typical TTS X-ray luminosities are $L_{\mathrm{X}} \sim 10^{30}-10^{31} \mathrm{erg} \mathrm{s}^{-1}$ (e.g., Grosso et al. 2000). The presence of disks does not seem to influence the TTS X-ray properties, but as TTS lose their disks while contracting towards the main sequence, they become more X-ray luminous, presumably because of the increased rotation velocity and resulting enhanced dynamo (Stelzer \& Neuhäuser 2001). X-ray surveys of star-forming area are very efficient to make a reliable census of the TTS population. Nevertheless IR follow-ups are needed to confirm the nature of the X-ray selected objects, and their young age.

- TTS X-ray variability is generalized. Peak X-ray luminosities may be as high as $L_{\mathrm{X} \text {,peak }} \sim 10^{32} \mathrm{erg} \mathrm{s}^{-1}$ or more. The light curves are strongly suggestive of flares. In the best cases, flare spectroscopy can be time-resolved, showing a 


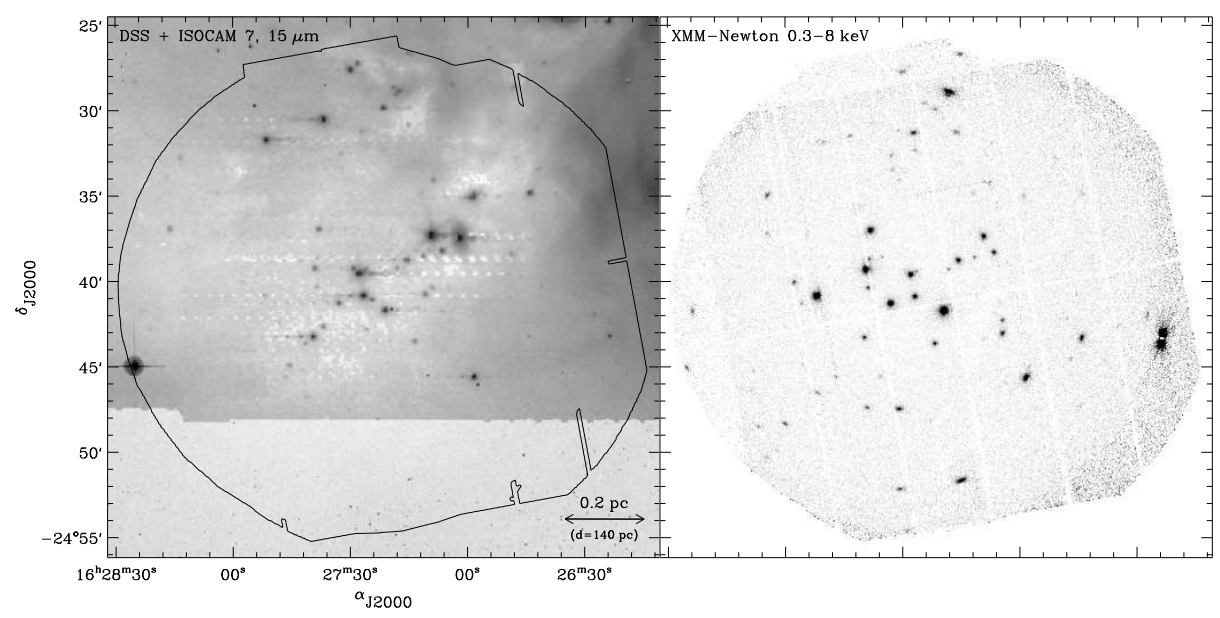

Fig. 3. Left: Field centered on the core F region of the $\rho$ Ophiuchi dark cloud. Background: DSS optical image. Foreground: ISOCAM $7+15 \mu \mathrm{m}$ image (Abergel et al. 1996). Quasi-circular contour: XMM-Newton/EPIC field-of-view. Most of the bright ISOCAM sources are protostars, the other sources are T Tauri stars (see Bontemps et al. 2001). Right: XMM-Newton image of the same region. With a few exceptions, almost all the IR sources, including protostars, are detected (Grosso et al., in preparation).

clear decline in temperature (from $T_{\mathrm{X}}$ as high as $\sim 10^{8} \mathrm{~K}$ down to a few $10^{7} \mathrm{~K}$ : see the example of V773 Tau, Tsuboi et al. 1998).

- X-ray detections have reached the brown dwarf limit. A few bona fide brown dwarfs have been detected in X-rays, already by ROSAT (Neuhäuser et al. 1999), and recently by Chandra (Rutledge et al. 2000; Imanishi et al. 2001) and XMM-Newton (Grosso et al., in preparation). Their $L_{\mathrm{X}} / L_{\mathrm{bol}}$ ratio is found to be $\gtrsim 10^{-4}$, i.e., very similar to that of TTS. It is reasonable to think that a number of new brown dwarf candidates will soon be $X$-ray detected among Chandra and $X M M-N e w t o n$ sources in star-forming regions. An IR spectroscopy confirmation will be necessary, and will require large telescopes.

- Deeply embedded sources like protostars are more difficult to detect in Xrays. The number of protostars detected remained small (about a dozen) in the ROSAT/ASCA era, for lack of access to the hard X-ray range (ROSAT), or limited sensitivity (ASCA) (FM). The new Chandra and XMM-Newton results on the $\rho$ Ophiuchi dark cloud (see above), are very promising, with a $\sim 70 \%$ detection rate, including at their non-flare, quiescent level.

- Almost all of the $\sim 15$ bona fide protostars detected so far in X-rays are evolved ("Class I") protostars. Although magnetism also plays a central role in their X-ray emission, significant differences exist with that of TTS : most detected Class I protostars have X-ray luminosities and temperatures somewhat higher than those of most TTS. Variability is as ubuquitous as for TTS, with many examples of flares. In a few cases $L_{\mathrm{X} \text {,peak }}$ reaches very high values of several $10^{32} \mathrm{erg} \mathrm{s}^{-1}$ or more, which may be due to star-disk magnetic interactions 
(Tsuboi et al. 2000, Montmerle et al. 2000). In contrast, the X-ray detection of young ("Class 0") protostars is open to discussion. Tsuboi et al. (2001) recently reported the Chandra discovery of two weak X-ray sources in the direction of candidate Class 0 protostars in the Orion OMC $2 / 3$ clouds, but their extinction, measured from the X-ray spectrum itself, is nearly one order of magnitude smaller than the typical extinction towards the central object of a Class 0 source across its own envelope. They may therefore be somehow associated with the Class 0 protostars, but not be strictly identified with them.

\section{The role of the VLT}

Thanks to their much improved sensitivity and resulting improved performance in spectral and spatial resolution, Chandra and XMM-Newton allow detailed studies of previously known, X-ray bright YSOs like TTS and protostars. But a new frontier appears: low-luminosity objects. With a few exceptions, the $L_{\mathrm{X}} / L_{\mathrm{bol}} \sim$ $10^{-4}$ correlation approximately holds empirically for all YSOs, so that in general weak X-ray sources (now detected at the level of $L_{\mathrm{X}} \sim 10^{28} \mathrm{erg} \mathrm{s}^{-1}$, i.e., only 10 times the active Sun, at $d \sim 150 \mathrm{pc}$ ) are expected to be intrinsically lowluminosity sources, i.e., of low mass and/or low temperature. Very Low-Mass objects and brown dwarfs are typical examples.

However, contrary to the situation holding with the past generation of Xray satellites, low-luminosity sources are not necessarily YSOs ! The reason is that the sensitivity of Chandra and XMM-Newton is such that weak, hard Xray sources like AGNs start to be visible through molecular clouds. From the extragalactic $\log N-\log S$ curve of the Hubble Deep Field seen by Chandra, the contamination is estimated to be $\approx 20$ background sources in the $17^{\prime} \times 17^{\prime}$ field of view (Garmire et al. 2001). Of course, such sources must be among the weakest and most absorbed of the sample, mimicking very low-mass stars. Note that the same situation holds in the IR, where background stars and galaxies routinely contaminate the young star sample, except in the densest regions.

It is therefore clear that one needs both X-ray and IR observations to disentangle the source sample, and large ground-based telescopes are mandatory in almost all cases. More generally, we have also seen (§2) the deep connection existing between the X-ray and IR ranges. Therefore, the VLT, with its large choice of IR cameras, is certainly the facility of choice for follow-ups of the sensitive $\mathrm{X}$-ray observations in progress.

A foretaste of the discoveries to come is offered by the preliminary results of an NTT/SOFI campaign to search for counterparts of XMM-Newton new X-ray sources without 2MASS counterpart in the $\rho$ Ophiuchi dark cloud (see Fig. 3; Grosso et al., in preparation). In the course of this study, two spectacular new embedded Herbig-Haro objects were found, one probably related to the peculiar X-ray emitting protostar YLW15, the other to the weaker X-ray emitting protostar, IRS54 (Grosso et al. 2001). The campaign has also yielded very low-luminosity IR counterparts for faint XMM-Newton sources, the nature of which can be elucidated only with follow-up IR spectroscopy such as provided 
by ISAAC in the near-IR or VISIR in the mid-IR. Could these be "free-floating planets" so well appreciated by Mark McCaughrean? (After all, Jupiter is also an X-ray source, although of auroral origin...)

\section{References}

1. Abergel, A., et al. 1996, A\&A, 315, L329

2. Audard, M., et al. 2001, A\&A, 365, L329

3. Bontemps, S. et al. 2001, A\&A, 372, 173

4. Casanova, S., Montmerle, T., Feigelson, E.D., \& André, P. 1995, ApJ, 439, 752

5. den Boggende, A. J. F., Mewe, R., Gronenschild, E. H. B. M., Heise, J. \& Grindlay, J. E. 1978, A\&A, 62, 1

6. Feigelson, E.D., \& Montmerle, T. 1999, Ann. Rev. Astr. Ap., 37, 363 [FM]

7. Friedman, H., Lichtman, S.W., \& Byram, E.T. 1951, Phys. Rev., 83, 1025

8. Garmire, G., Feigelson, E. D., Broos, P., Hillenbrand, L. A., Pravdo, S. H., Townsley, L., \& Tsuboi, Y. 2000, AJ, 120, 1426

9. Garmire, G., et al. 2001, ApJ, submitted

10. Giacconi, R., Murray, S., Gursky, H., Kellogg, E., Schreier, E. \& Tananbaum, H. 1972, ApJ, 178, 281

11. Glassgold, A.E., Feigelson, E.D., \& Montmerle, T. 2000, in Protostars \& Planets $I V$, Eds. V. Mannings, A. Boss, \& S. Russell (Tucson: U. of Arizona Press), p. 429 [GFM]

12. Grosso, N. 2001, A\&A, 370, L22

13. Grosso, N., Alves, J., Neuhäuser, R. \& Montmerle, T. 2001, A\&A, submitted

14. Grosso, N., Montmerle, T., Feigelson, E. D., et al. 1997, Nature, 387, 56

15. Grosso, N., Montmerle, T., Bontemps, S., André, P. \& Feigelson, E.D. 2000, A\&A, 359,113

16. Imanishi, K., Koyama, K. \& Tsuboi, Y. 2001, ApJ, 557, 747

17. Ku, W.H. \& Chanan, G.A. 1979, ApJ, 234, L59

18. Montmerle, T., Grosso, N., Tsuboi, Y. \& Koyama, K. 2000, ApJ, 532, 1097

19. Neuhäuser, R., Briceño, C., Comerón, F., Hearty, T., Martín, E. L., Schmitt, J. H. M. M., Stelzer, B., Supper, R., Voges, W. \& Zinnecker, H. 1999, A\&A, 343, 883

20. Rutledge, R. E., Basri, G., Martín, E. L., \& Bildsten, L. 2000, ApJ, 538, L141

21. Ryter, C.E. 1996, Astr. Sp. Sci., 236, 285

22. Sciortino, S. 2001, in From Darkness to Light, Eds. T. Montmerle \& P. André, ASP Conf. Ser. 243, in press

23. Stelzer, B. \& Neuhäuser, R.N. 2001, A\&A, in press

24. Tsuboi, Y., Koyama, K., Murakami, H., Hayashi, M., Skinner, S., \& Ueno, S. 1998, ApJ, 503, 894

25. Tsuboi, Y., Imanishi, K., Koyama, K., Grosso, N., \& Montmerle, T. 2000, ApJ, 532, 1089

26. Tsuboi, Y., Koyama, K., Hamaguchi, K., Tatematsu K., Sekimoto, Y., Bally, J. \& Reipurth, B. 2001, ApJ, 554, 734

27. Wilms, J., Allen, A., \& McCray, R. 2000, ApJ, 542, 914 